\title{
Comparación de Modelos No Lineales para Describir Curvas de Crecimiento según Sexo en el Borrego Chiapas
}

\author{
Comparison of Non-Linear Models for Describing Growth Curves According \\ to SeX in Ovine Chiapas B ReEd
}

\author{
Pere Miquel Parés-Casanova ${ }^{1,3}$, Marta Caballero', Raúl Perezgrovas²
}

\section{Resumen}

El presente trabajo tuvo por objetivo estudiar las curvas de crecimiento de machos y hembras del Borrego Chiapas. Se evaluó la capacidad de ajuste de tres modelos de crecimiento: logístico, von Bertalanffy y Gompertz, a los datos de peso de 66 machos y 79 hembras de diversa edad. Los tres modelos presentaron valores muy próximos en cuanto a los criterios de bondad de ajuste, donde el modelo logístico es el que mejor describe las curvas de crecimiento en el Borrego Chiapas, y puede ser utilizado para estudiar la cinética del crecimiento en esta raza en los dos sexos. Aun así, el modelo logístico tiende a sobrestimar el peso inicial de los animales.

Palabras clave: modelo logístico, modelo von Bertalanffy, modelo Gompertz, ontogenia

\section{AbStract}

The aim of this study was to establish the growth curves in ovine Chiapas breed according to sex. Three models were used: logistic, von Bertalanffy and Gompertz. Body weight data were obtained from 66 males and 79 females of various ages. The three models showed similar goodness values; however, the logistic model was the best to describe the growth kinetics in this breed for both males and females, although this model tends to overestimate the initial body weights.

Key words: logistic model, von Bertalanffy model, Gompertz model, ontogeny

\footnotetext{
${ }^{1}$ Departament de Producció Animal, Universitat de Lleida, Lleida, Catalunya, España

${ }^{2}$ Laboratorio de Calidad de Lana, Instituto de Estudios Indígenas, Universidad Autónoma de Chiapas, San Cristóbal de Las Casas, Chiapas, México

${ }^{3}$ E-mail: peremiquelp@prodan.udl.cat
}

Recibido: 11 de enero de 2015

Aceptado para publicación: 30 de marzo de 2015 


\section{INTRODUCCIÓN}

Los modelos matemáticos son ecuaciones que, entre otras cosas, permiten construir curvas continuas de una variable biológica en función de otra. Esta información es importante para efectos de investigación y recomendaciones de orden zootécnico (p. ej. buscando precocidad y mejor calidad de la canal (Souza y Bianchini, 1994). Diferentes modelos han sido utilizados para describir la relación entre la edad del animal, su velocidad de crecimiento y madurez, pero dado que el crecimiento animal no sigue una tendencia lineal, es necesario explorar modelos no lineales que permitan estudiar este parámetro.

Las relaciones entre el incremento de una dimensión en función del tiempo pueden expresarse mediante las curvas generales de crecimiento. Como es sabido, estas curvas tienen una forma de tipo sigmoideo cuando el incremento de la dimensión se manifiesta en términos absolutos. Los modelos matemáticos utilizados para modelar el crecimiento animal tienen forma sigmoidal, pudiéndose diferenciar en ellos las siguientes fases: 1. Fase de aceleración, que idealmente debe tener su origen en el punto (0.0), y se caracteriza porque la velocidad de crecimiento es muy rápida y positiva llegando al máximo en el punto de inflexión de la curva; 2. Fase de desaceleración, en la que a partir del punto de inflexión la tasa de crecimiento comienza a disminuir; y 3. Fase lineal, en la que el animal deja de crecer o el crecimiento puede ser considerado como una mera reposición de tejidos.

Los modelos logístico, de Gompertz y de von Bertalanffy (von Bertalanffy, 1976a,b,c) son algunas de las funciones de crecimiento frecuentemente utilizadas para describir el crecimiento de plantas, animales y órganos. Son clásicas las aplicaciones de estos métodos en investigaciones pesqueras, aunque se han utilizado también satisfactoriamente en el estudio del crecimiento de di- mensiones ponderales y lineales en los mamíferos. Estos modelos (Cuadro 1) presentan tres parámetros con interpretación biológica y uno definido como constante matemática. El parámetro $a$ corresponde al peso asintótico o peso adulto a la madurez. El parámetro $c$ corresponde a la estimativa de precocidad de madurez (Nobre et al., 1987); cuanto mayor sea el valor de este parámetro más precoz es el animal y viceversa (Brown et al., 1976). El parámetro $b$ es denominado parámetro de integración, y no posee significado biológico.

El Borrego Chiapas es un animal rústico, cuya crianza está orientada a la producción de lana, y está muy bien adaptado a las zonas montañosas del oeste de México. Es una de las razas más importantes del país en cuanto a producción de lana y solo es utilizado por este fin debido a la prohibición de su consumo entre los indígenas tzotziles (Perezgrovas, 2004). La ovinocultura indígena en la región montañosa de Los Altos de Chiapas difiere con la practicada en otras partes de México, pues se aleja de los patrones establecidos ya que es desarrollada exclusivamente por mujeres tzotziles. Ellas se encargan del cuidado de las ovejas, de la esquila y del tejido de la lana para la confección de sus vestidos tradicionales, tomando las decisiones relacionadas con esta actividad que, además de productiva, tiene un carácter socio-cultural (Perezgrovas, 2004).

El objetivo de este trabajo fue evaluar los modelos matemáticos logístico, von Bertalanffy y Gompertz, en cuanto a su capacidad para describir el crecimiento del Borrego Chiapas.

\section{Materiales y Métodos}

Se utilizaron 66 machos y 79 hembras del Borrego Chiapas de diversas edades (rango: 3 a 156 meses), procedentes de varias comunidades de la zona de Chiapas, México. Se registró la edad en base a las declaracio- 
Cuadro 1. Descripción matemática de los modelos no lineales de crecimiento para la evaluación del crecimiento del Borrego Chiapas

\begin{tabular}{lcl}
\hline Modelo & Número de parámetros & Expresión matemática \\
\hline Logístico & 3 & $\mathrm{y}=\mathrm{a} /\left(1+\mathrm{be}^{-\mathrm{cx}}\right)$ \\
Gompertz & 3 & $\mathrm{y}=\mathrm{a} * \exp \left(\mathrm{b}^{*} \exp (\mathrm{cx})\right.$ \\
von Bertalanffy & 3 & $\mathrm{y}=\mathrm{a}\left(1-\mathrm{be} \mathrm{e}^{-\mathrm{cx}}\right)$ \\
\hline $\mathrm{y}=$ peso del animal en el tiempo & \\
$\mathrm{a}=$ estimativa del peso a la madurez \\
$\mathrm{b}=$ parámetro de integración, no posee significado biológico \\
$\mathrm{c}=$ estimativa de precocidad de madurez
\end{tabular}

nes de los ganaderos, y el peso vivo se obtuvo mediante el pesado directo de los animales con balanza digital. Todos los datos fueron recabados por la misma persona.

Los modelos utilizados para describir el crecimiento de los animales fueron el logístico, el de Gompertz y el de von Bertalanffy. Las expresiones matemáticas que representan a cada uno de los modelos y su número de parámetros son presentados en el Cuadro 1. Los modelos fueron ajustados a la edad de cada animal utilizando el procedimiento para modelizaciones no lineales del programa PAST (Hammer et al., 2010). Para distinguir la capacidad de ajuste de cada uno de los modelos en estudio fue utilizado el criterio de información de Akaike (AIC), que combina teoría de máxima verosimilitud, información teórica y entropía de información (Motulsky y Christopoulos, 2003), considerando el mejor modelo aquel de menor valor AIC. La comparación del modelo según sexo se realizó con el test ANCOVA, previa transformación logarítmica de los datos.

\section{Resultados y Discusión}

Los valores AIC de los modelos en estudio se presentan en el Cuadro 2. El menor valor de AIC para machos y hembras se observó en el modelo logístico, con valores de
3549.9 y 2043.8 , respectivamente; sin embargo, pese a que este modelo presentó los menores valores, la variación entre modelos para el ajuste de los datos fue muy estrecha.

Además de los criterios matemáticos que permiten determinar la capacidad de ajuste de un modelo frente a otro, se requiere tener en cuenta como parámetro de evaluación la coherencia biológica de los parámetros estimados del modelo. En el Cuadro 3 se presentan los valores de los parámetros ecuacionales y la amplitud de la variación de los parámetros de las curvas individuales de crecimiento para los ovinos de ambos sexos después de su ajuste al modelo logístico.

En las figuras 1 y 2 se presentan las curvas de crecimiento observadas y predichas por el modelo logístico para machos y hembras, respectivamente. El test ANCOVA reflejó que no existían diferencias estadísticamente significativas de crecimiento entre sexos $(\mathrm{F}=0.575, \mathrm{p}=0.450)$. Puede observarse que el modelo logístico tiende, en ambos sexos, a sobreestimar el peso inicial.

El modelo reveló que los machos presentan un mayor peso maduro que las hembras. La estimativa del peso maduro (parámetro $a$ ) fue mayor para los machos. Animales con un alto valor de $c$ presentan madurez precoz en comparación con indivi- 
Cuadro 2. Valores del criterio de información de Akaike (AIC) de los modelos en estudio

\begin{tabular}{llc}
\hline Modelo & Sexo & AIC \\
\hline Logístico & Machos & 3549.9 \\
& Hembras & 2043.8 \\
Gompertz & Machos & 3703.2 \\
& Hembras & 2130.2 \\
\multirow{2}{*}{ von Bertalanffy } & Machos & 4059.5 \\
& Hembras & 2308.2 \\
\hline
\end{tabular}

Cuadro 3. Estimativa de los parámetros de la función de crecimiento del modelo logístico

\begin{tabular}{lcll}
\hline Sexo & Parámetro & Valor & IC $^{1} 95 \%$ \\
\hline Machos & .a & 40.04 & 40.04 a 45.05 \\
& .b & 1.005 & 0.692 a 1.493 \\
Hembras & .c & 0.006 & -0.01 a 0.01 \\
& .a & 31.03 & 31.03 a 33.03 \\
& .b & 1.137 & 0.351 a 1.519 \\
& .c & 0.019 & -0.0007 a 0.02 \\
\hline
\end{tabular}

${ }^{1}$ Intervalo de confianza

duos de valores más bajos de $c$ y de peso inicial semejante. Las tasas de crecimiento expresadas en gramos día ${ }^{-1}$ estimadas por el modelo son menores para machos que para hembras, siendo una diferencia apreciable (casi del doble). Por ello, se deduce que los machos crecen a una tasa menor que las hembras, alcanzando estas, a la misma edad, un peso adulto menor. De todos modos, cabe decir que aunque los registros de pesaje de estos animales se llevaron hasta los 156 meses después del nacimiento, fue imposible establecer con claridad la fase asintótica de la curva.

Según Hammond (1932), en un organismo que crece se operan dos tipos de fenómenos: a) el incremento en peso y volumen en el transcurso del tiempo, y b) la modificación de las proporciones de las diversas re- giones morfológicas, órganos y sistemas, hasta que se accede al estado adulto o estable. Para el Borrego Chiapas, el modelo logístico presenta un patrón de crecimiento similar para ambos sexos, aunque el incremento general del peso es mayor para las hembras.

El grado de dimorfismo sexual depende de muchos factores (Frayer y Wolpoff, 1985), pero uno de los principales se relaciona a la nutrición proteico-calórica, que en caso de ser inadecuada actúa reduciendo las diferencias en tamaño entre sexos (Pucciarelli, 1980). En casos de estrés nutricional y, por lo menos, en la especie humana, los hombres son más susceptibles, por lo que reducen su tamaño, mientras que las mujeres son más estables (Stini, 1969). Esto podría explicar las diferencias en el desarrollo de los ovinos de Chiapas dadas las condiciones nutricionales 


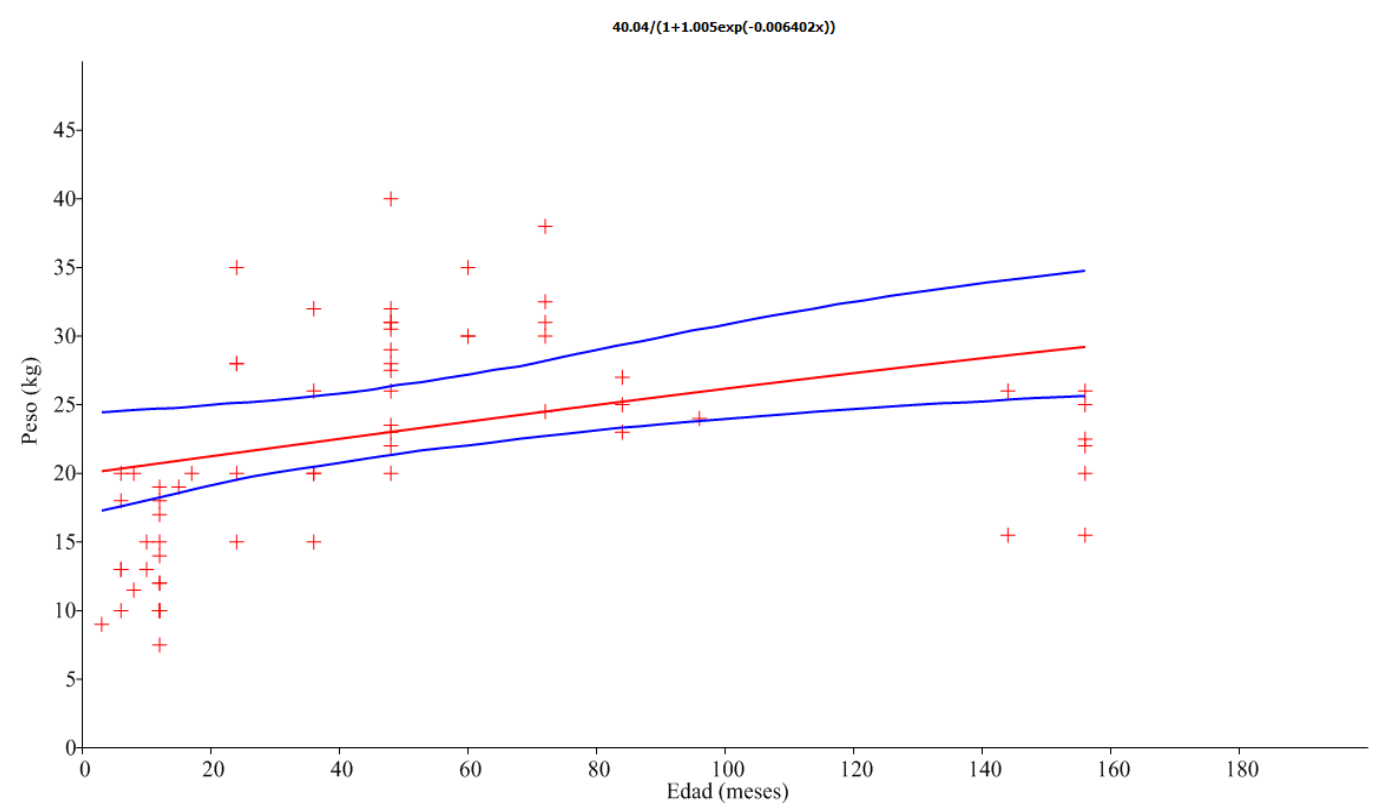

Figura 1. Curvas de crecimiento observadas y predichas para Borregos Chiapas machos (modelo logístico). El trazo azul indica el intervalo de confianza del 95\%

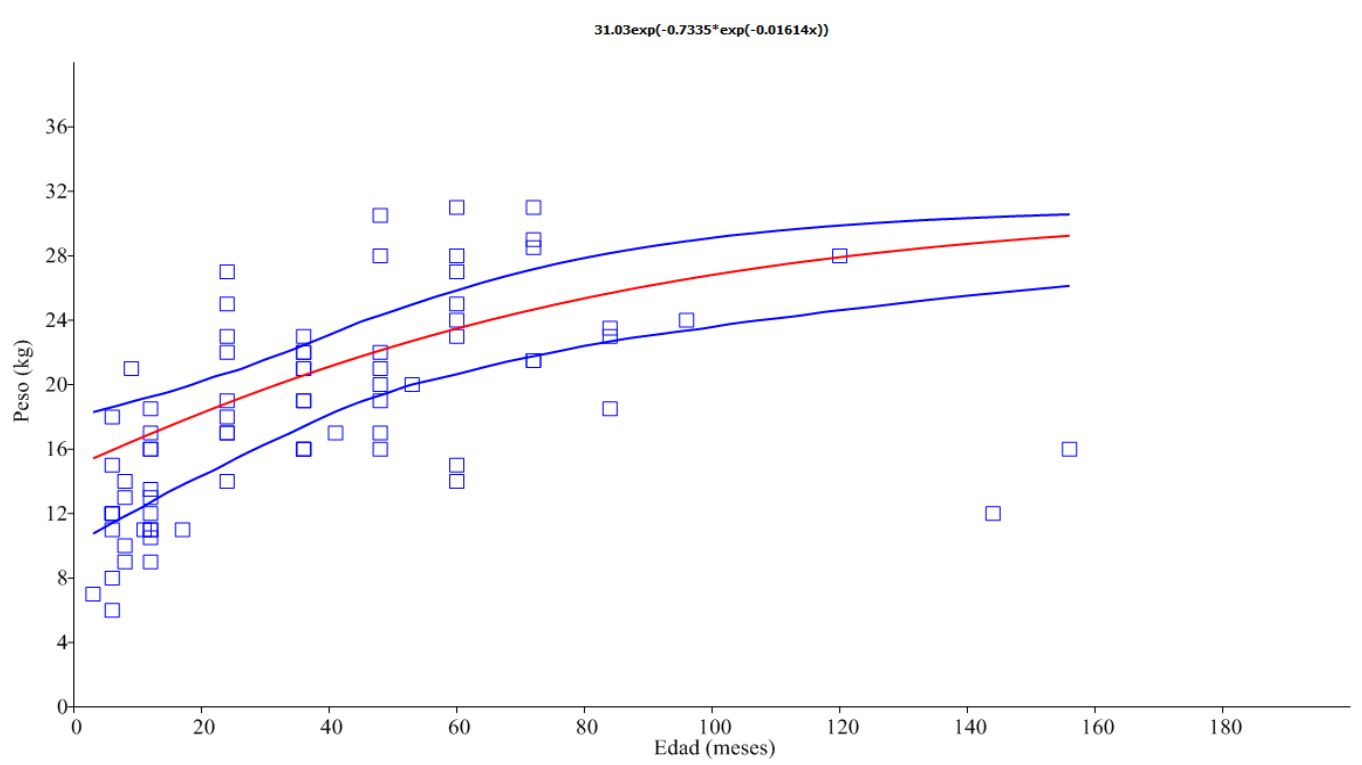

Figura 2. Curvas de crecimiento observadas y predichas para Borregos Chiapas hembras (modelo logístico). El trazo azul indica el intervalo de confianza del 95\% 
subóptimas existentes. Análisis histológicos y osteológicos, fuera del alcance de este estudio, podrían aportar mejor información para confirmar o rechazar esta hipótesis.

En síntesis, el presente trabajo aporta algunos elementos para comprender un patrón de crecimiento ligeramente diferente en machos y hembras del Borrego Chiapas. Se concluye que las pocas diferencias existentes a lo largo del crecimiento para ambos sexos podrían relacionarse con deficiencias nutricionales, siendo este retraso especialmente marcado para los machos.

\section{Literatura Citada}

1. Anzelmo M, Sardi ML, Barbeito-Andrés J, Pucciarelli HM. 2012. Alometrías ontogénicas y dimorfismo sexual facial en dos poblaciones humanas modernas. Rev Arg Antropol Biol 14(1): 89-100.

2. Bertalanffy von L. 1976a. El organismo considerado como sistema físico. En: Teoría general de los sistemas. México: Fondo de Cultura Económica. p. 124-143.

3. Bertalanffy von L. 1976b. El modelo del sistema abierto. En: Teoría general de los sistemas. México: Fondo de Cultura Económica. p. 144-160.

4. Bertalanffy von L. 1976c. Algunos aspectos de la teoría de los sistemas en biología. En: Teoría general de los sistemas. México: Fondo de Cultura Económica. p. 161-194.

5. Brown JE, Fitzhugh HA, Cartwright TC. 1976. A comparison of nonlinear models for describing weight-age relationships in cattle. J Anim Sci 42: 810818. doi:10.2134/jas 1976.424810x
6. Frayer DW, Wolpoff MH. 1985. Sexual dimorphism. Annu Rev Anthropol 14: 429-473. doi: 10.1146/annurev. an.14. 100185.002241

7. Hammer $\emptyset$, Harper DAT, Ryan PD. 2010. PAST: Paleontological statistics software package for education and data analysis. v. 2.17c. Palaeontol Electron 4(1). [Internet]. http://palaeo-electronica.org/2001_1/past/past.pdf

8. Hammond J. 1932. Growth and the development of mutton qualities in the sheep. London: Oliver \& Boyd. 597 p.

9. Motulsky H, Christopoulos A. 2003. Fitting models to biological data using linear and nonlinear regression, v. 4. GraphPad Software. [Internet]. Disponible en: http://www.graphpad.com/ manuals/prism4/RegressionBook.pdf

10. Nobre PRC, Rosa A, Silva LO, Evangelista SR. 1987. Curvas de crescimento de gado Nelore ajustadas para diferentes frequências de pesagens. Pesq Agropeo Bras 22: 1027-1037.

11. Perezgrovas Garza R. 2004. Los carneros de San Juan: ovinocultura indígena en Los Altos de Chiapas. $3^{a}$ ed. México: Universidad Autónoma de Chiapas. $374 \mathrm{p}$.

12. Pucciarelli HM. 1980. The effect of race, sex and nutrition on craniofacial differentiation in rats. A multivariate analysis. Am J Phys Anthropol 53: 359368.

13. Souza JC, Bianchini E. 1994. Estimativa do peso de bovinos de corte, aos 24 meses, da raça Nelore, usando curvas de crescimento. Rev Soc Bras Zootec 23: 85-91.

14. Stini WA. 1969. Nutritional stress and growth: sex difference in ada ptive response. AmJ Phys Anthropol 31:417-426. 